\title{
Acute renal injury induced by valacyclovir hydrochloride: A case report
}

\author{
YANNING ZHANG, YUXI CONG and YAN TENG \\ Department of Nephrology, People's Liberation Army No. 202 Hospital, Shenyang, Liaoning 110000, P.R. China
}

Received July 15, 2015; Accepted September 6, 2016

DOI: $10.3892 /$ etm.2016.3905

\begin{abstract}
Acyclovir has been a frequently used antiviral agent in the clinical treatment of leukemia, acute encephalitis, malignant tumor and herpes simplex. The adverse effects of this drug have been widely described in clinical practice. In the present study, a case of a 35-year-old female patient diagnosed with herpes simplex, who developed acute renal injury following treatment with valacyclovir hydrochloride, is described. Kidney biopsy, light microscopy and laboratory examination were performed, and all findings revealed the signs of evident vacuolar degeneration of capillary endothelial and renal tubular epithelial cells, erythrocyte aggregation in partial renal tubule and microvilli exfoliation from epithelial cells. Renal interstitial edema was clearly identified. The clinical evidence observed from this female patient indicated that renal functions should be closely monitored during valacyclovir hydrochloride administration. A variety of effective measures, such as hydration, alkalizing urine, promoting the discharge of medication and the use of antagonists are recommended following the administration of antiviral agents.
\end{abstract}

\section{Introduction}

Valacyclovir hydrochloride is an antiviral drug used in the management of herpes simplex, herpes zoster and herpes B. It is a prodrug and can be rapidly converted into acyclovir in vivo (1-3). In addition to neurotoxicity, acute kidney injury is a well-described side effect of acyclovir administration, since crystal deposition may lead to the development of renal failure $(4,5)$. The side-effects of acyclovir therapy are not well recognized by clinicians.

Linssen-Schuurmans et al (6) first reported valacyclovir associated neurotoxicity in 1998, and more cases have been

Correspondence to: Dr Yanning Zhang, Department of Nephrology, People's Liberation Army No. 202 Hospital, 5 Guangrong Road, Shenyang, Liaoning 110000, P.R. China

E-mail: yanningzhang123@yeah.net

Key words: acute renal injury, herpes simples, acyclovir, adverse event reported cases since then. Asahi et al (7) reviewed 20 cases of chronic renal failiue, and both of the other 3 cases, without previous renal failure, had acute renal failure when valacyclovir neurotoxicity appeared. Three of the 17 patients (17.6\%) had received valacyclovir irregularly, hence their actual dosage was uncertain; but of the other 14 patients, 8 patients $(57.1 \%)$ had clearly received excessive doses. Adair et al (8) reviewed 30 cases of acyclovir neurotoxicity. Seven of them had no renal insufficiency before receiving acyclovir. Acyclovir can produce kidney failure either through precipitation within the tubular lumen or from acute interstitial nephritis. With the extensive clinical application of acyclovir, adverse drug reactions, especially acute renal injury, have rapidly increased, which were reported by Fleischer et al (4) and Obada et al (9).

Acute kidney injury secondary to acyclovir is characterized by a decrease in renal function that typically develops within a certain period of time, usually $12-48 \mathrm{~h}$ following drug administration, as indicated by a rapid elevation in the serum creatinine $(5,10)$. Immediate detection of acute kidney injury is necessary to prevent the progression and aggravation of renal diseases (11-14). The current study reviews the clinical features, diagnosis and management of acyclovir nephrotoxicity, aiming to add clinical evidence to early diagnosis and treatment of acyclovir-induced acute renal injury.

\section{Case report}

Baseline data. A female patient, aged 35 years, was hospitalized due to complaints of a hip blister for 6 days, fever enduring for 2 days and kidney dysfunction for 1 day (People's Liberation Army No. 202 Hospital, Shenyang, China) in January 2014. The patient was admitted to another local hospital and diagnosed with herpes simplex due to the hip blister with unknown causes accompanied by slight erythema. The patient was administered with valacyclovir hydrochloride (dosage unknown) and then presented with lumbar pain. At 2 days prior to admission, the patient had fever accompanied by shiver at $37.8^{\circ} \mathrm{C}$ body temperature. Additionally, the patient self-reported pain in the lower abdomen, and orally administered Pudilan anti-inflammatory oral liquid combined with the external use of iodophor solution. The patient's body temperature was lowered to $36.9^{\circ} \mathrm{C}$, whereas the lumbar discomfort was not evidently improved; however, dysuria and hypourocrinia were experienced, and the daytime quantity of urine 
output was $\sim 400 \mathrm{ml}$. Subsequently, the patient was admitted to our hospital for kidney dysfunction examination.

Comprehensive examinations. Serum creatinine was increased to $592.7 \mu \mathrm{mol} / 1 \mathrm{l}(\uparrow)$ and uric acid was increased to $624 \mu \mathrm{mol} / \mathrm{l}$ $(\uparrow)$. Physical examination revealed a body temperature of $36.9^{\circ} \mathrm{C}$, pulse of 70 times $/ \mathrm{min}$, respiration rate of 18 times $/ \mathrm{min}$ and blood pressure of $121 / 74 \mathrm{mmHg}$. No abnormality was noted in the heart, lung and abdomen. No percussion pain was detected in the bilateral kidney. No tenderness pain was found in the hypochondrium, upper and medial ureter, costovertebral angle and costolumbar points. No percussion pain was noted in the costovertebral angle. Neither bulge nor tenderness was identified in the suprapubic space. No mass or edema was detected in bilateral lower extremities. Clusters of erythema and blister with clear margin were observed in the hip. Laboratory examination revealed a white blood cell count of $8.6 \times 10^{9} / 1\left(4-10 \times 10^{9} / 1\right)$, neutrophilic leukocyte percentage of $80.4 \%(0.5-0.7 \%)$, eosinophil percentage of $2.1 \%$ (0.005-0.05\%), red blood cell count of $4.2 \times 10^{12} / 1\left(3.5-5 \times 10^{12} / 1\right)$ and hemoglobin concentration of $127 \mathrm{~g} / 1$ (110-150 g/l). Renal function tests revealed a serum creatinine level of $592.7 \mu \mathrm{mol} / 1$ (41-73 $\mu \mathrm{mol} / \mathrm{l})$, urea nitrogen concentration of $15.37 \mathrm{mmol} / \mathrm{l}$ (2.6-7.5 mmol/1), Cystatin C of $2.14 \mathrm{mg} / 1$ (0.6-1.4 mg/l), blood uric acid of $624 \mu \mathrm{mol} / 1(90-420 \mu \mathrm{mol} / \mathrm{l})$, total immunoglobulin ( $\mathrm{Ig}) \mathrm{E}$ of $28.9 \mathrm{mg} / \mathrm{l}(0.1-0.9 \mathrm{mg} / \mathrm{l})$ and C-reactive protein of $33.60 \mathrm{mg} / 1$ (0-3 mg/l). Routine urine examination revealed a urine specific gravity of $1.0100(\downarrow)$, occult blood $(+++)$ and urine protein $(-)$; microscopic examination of red blood cells revealed 1-3/HP, white blood cells (-), urine IgG of $18.80 \mathrm{mg} / \mathrm{l}(\uparrow)$, urine trace albumin of $57.30 \mathrm{mg} / \mathrm{l}(\uparrow)$, urine $\alpha 1$ microglobulin of $21.10 \mathrm{mg} / \mathrm{l}(\uparrow)$ and urine transferrin of $3.280 \mathrm{mg} / \mathrm{l}(1.9-31 \mathrm{mg} / \mathrm{l})$. Further tests revealed blood potassium of $4.2 \mathrm{mmol} / \mathrm{l}(3.5-5.3 \mathrm{mmol} / \mathrm{l})$, sodium of $136 \mathrm{mmol} / \mathrm{l}$ (135-145 mmol/l), calcium of $2.25 \mathrm{mmol} / \mathrm{l}(2.12-2.75 \mathrm{mmol} / \mathrm{l})$, B-type natriuretic peptide of $1,890 \mathrm{pg} / \mathrm{ml}(\uparrow)$, erythrocyte sedimentation rate of $51 \mathrm{~mm} / \mathrm{h}(\uparrow)$, fibrinogen of $4.31 \mathrm{~g} / 1 \uparrow$, inorganic phosphorus of $2.01 \mathrm{mmol} / 1 \uparrow$, HBsAg positive, $\mathrm{HBcAb}$ positive $(\uparrow)$, quantification of hepatitis B virus $<500 \mathrm{IU} / \mathrm{ml}$, antinuclear antibody detection and antineutrophil cytoplasmic antibody detection were negative and ultrasound examination of bilateral kidneys revealed no renal abnormality. Pulmonary computed tomography showed lung marking enhancement, multiple lesions in the bilateral lungs and multiple calcified lesions in the abdomen. Renal biopsy under light microscopy detected 27 glomeruli and no signs of glomeruli or segmental sclerosis. Slight hyperplasia of glomeruli mesangial cell and matrix was detected. No significant thickening of the basement membrane was noted. Neither epithelial cell hyperplasia nor crescent formation was documented. Mild fibrosis was found adjacent to glomeruli sacculus. Renal tubular epithelial cell granule and vacuolar degeneration were observed. Lumen ectasia was detected in partial renal tubules with absence of brush border. Renal interstitial edema was noted. Vessel wall thickening of the arteriole and lumen narrowing were noted. Immunofluorescent analysis revealed 7 glomeruli, negative outcomes for IgG, IgM, IgA, C3 and C1q. No marked thickening was noted in the glomeruli sacculus wall. No evidence of hyperplasia in parietal layer cells was detected. No significant thickening of the basement membrane was seen with
250-350 $\mu \mathrm{m}$ in thickness (Fig. 1). Epithelial cell swelling and vacuolar degeneration were noted. No apparent hyperplasia or dense deposit was found in mesangial cell paralinin. Renal tubular epithelial cell vacuolar degeneration, partial lumen ectasia, microvilli exfoliation from epithelial cells (Fig. 2A) and renal interstitial edema were noted (Fig. 2B and C). Evident vacuolar degeneration of capillary endothelial cells, erythrocyte aggregation in the partial lumen and opening of the capillary loop were observed (Fig. 2D). The findings of light microscope, immunofluorescence and electron microscopy were consistent with acute renal tubular injury. The patient was eventually diagnosed with acute renal injury and drug-induced nephropathy.

Clinical treatment. The patient was injected with glutathione (Chongying Yaoyou Pharmaceutical Co., Ltd., Chongqing, China) to alleviate renal tubular injury and sodium bicarbonate to cause alkalized urine to prevent the turbular formation. Then, the patient was injected with azithromycin (Northwest Pharmaceutical Co., Ltd., Shenyang, China) to treat cutaneous infection. Phenolsulfonic acid calcium capsule (0.25 g x 48; Ningxia Kangya Pharmaceutical Co., Ltd., Yinchuan, China) was supplemented to improve kidney circulation and accelerate the repairing of renal tubular epithelial cells. Compound polymyxin and topical use of iodophor (Zhejiang Ri Sheng Chang Pharmaceutical Co., Ltd., Dongyang, China) were delivered to manage herpes simplex. Upon discharge, the quantity of urine was significantly increased to $>3,000 \mathrm{ml} /$ day, serum creatinine levels were $76 \mu \mathrm{mol} / 1$, microglobulin levels were $5.90 \mathrm{mg} / 1$ and urine transferrin levels were $2.210 \mathrm{mg} / \mathrm{l}$. Routine urine examination revealed occult blood $( \pm)$, urine protein $(-)$, white blood cell 1-3/HP and red blood cell 6-8/HP. Follow-up for 3 months revealed the normal renal function.

\section{Discussion}

Valacyclovir hydrochloride is an antiviral drug used in the management of herpes simplex, herpes zoster and herpes B. Valacyclovir hydrochloride is a prodrug and can be rapidly converted into acyclovir in vivo (1-3). In addition to neurotoxicity, acute kidney injury is a well-described side effect of acyclovir administration, since crystal deposition may lead to the development of renal failure. In recent years, valacyclovir has largely replaced acyclovir in the treatment of herpes virus infections, because it is more effective by oral administration (7). There are several cases about the acute kidney injury caused by acyclovir reported, but the side effects of valacyclovir are not well recognized by clinicians, thus are rarely reported compared with acyclovir. Sagawa et al (15) found an elderly diabetic patient treated with valacyclovir was diagnosed with acyclovir-induced neurotoxicity and acute kidney injury later, although the patient with no microalbuminuria and a serum creatinine level seven days before admission, the age, metabolic disorder maybe the risk factors of the injury. In the present report, the patient is a young female without any disease history, and it has more important clinical significance to identify the possible side effects of valacyclovir.

Acyclovir, which is relatively insoluble in urine, is rapidly filtered by the glomeri and secreted by the renal tubules, which can produce high urine concentrations, particularly 


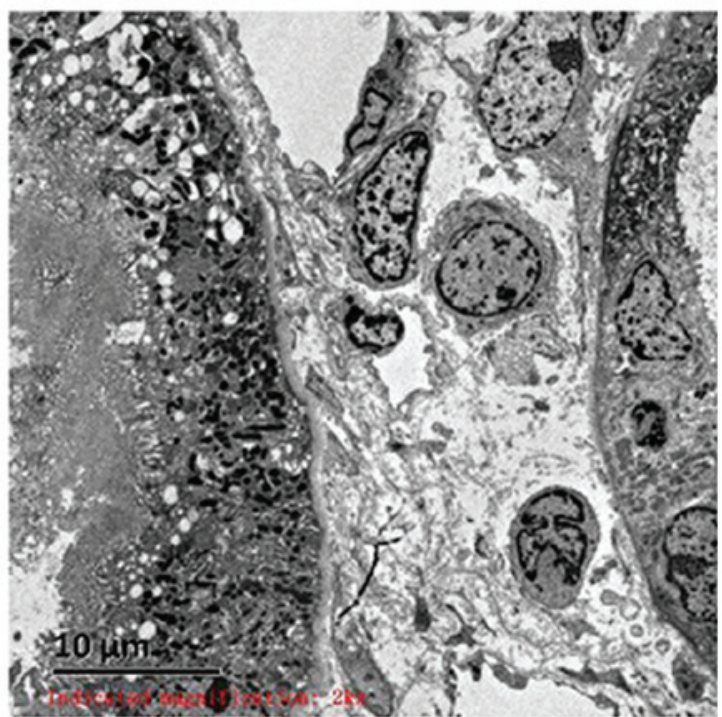

Figure 1. Electron microscopy of the patient's kidney.

in patients with decreased urine flow rates (16-19). Renal excretion accounts for 60-90\% of acyclovir elimination (20). The present study reports a case of a patient developing acute kidney injury secondary to herpes simplex after receiving acyclovir. Other potential mechanisms of injury include acute interstitial nephritis and acute tubular necrosis. However, the most commonly reported mechanism is obstructive nephropathy (20).

The use of acyclovir can damage the kidney via a number of mechanisms. Firstly, the severity of renal injury induced by acyclovir is associated with the dose used (10). It directly causes cell membrane injury, alters the membrane permeability and ion transportation. In addition, it destroys cytoplasm mitochondria, inhibits enzymatic activity and protein synthesis (21), promotes calcium internal flow and leads to cytoskeleton structural damage and epithelial cell necrosis. Furthermore, it is able to produce oxygen free radicals. It can also affect epithelial cell DNA, induces crosslinking or inhibits DNA replication of related enzymes and suppresses renal tubular epithelial cell metabolism. Secondly, acyclovir is primarily discharged from the urine and is almost insoluble in the urine, forms crystals and occludes the renal tubule (22). Thirdly, it is mediated by a variety of immune factors (22). Fourthly, it may cause thrombotic microangiopathy (23). Fifthly, the physiological nature of the kidney is highly susceptible to drug-induced nephropathy (21). The abundant renal capillary network and rich blood allow for high concentrations of drugs passing through a large contacted area. The counter-current multiplication mechanism also contributes to elevated drug concentration in the medulla renis. Furthermore, the variation in urine $\mathrm{pH}$ is likely to cause crystals and drug sediment in the renal tubule. The enzymes contained in the kidney would be deactivated by the medication. In the current report, the patient presented with valacyclovir hydrochloride-induced nephropathy, which has been rarely reported in clinical practice.

The female patient was orally administered with valacyclovir hydrochloride tablets to treat herpes simplex. Subsequently, the patient experienced a significant reduction in quantity of urine produced, and an apparent elevation in
A

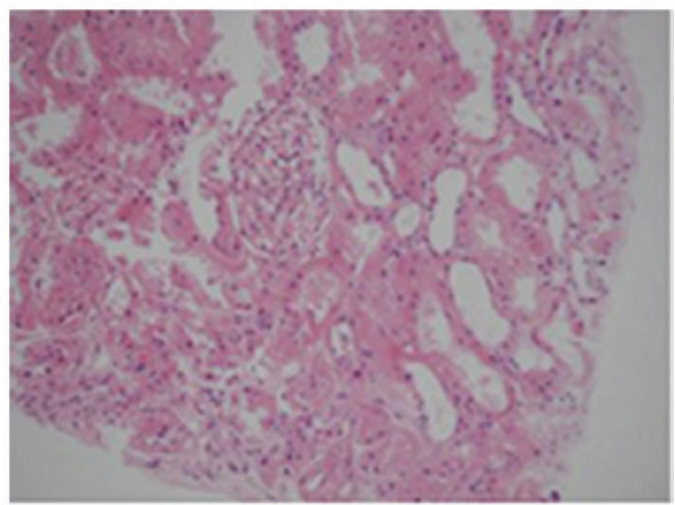

B

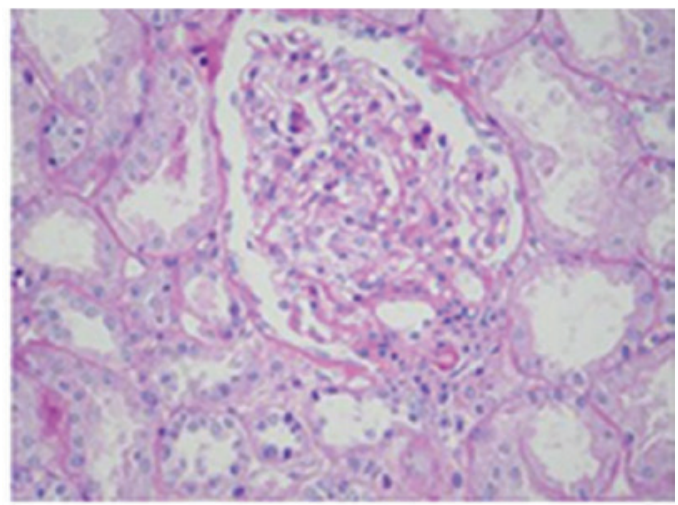

C

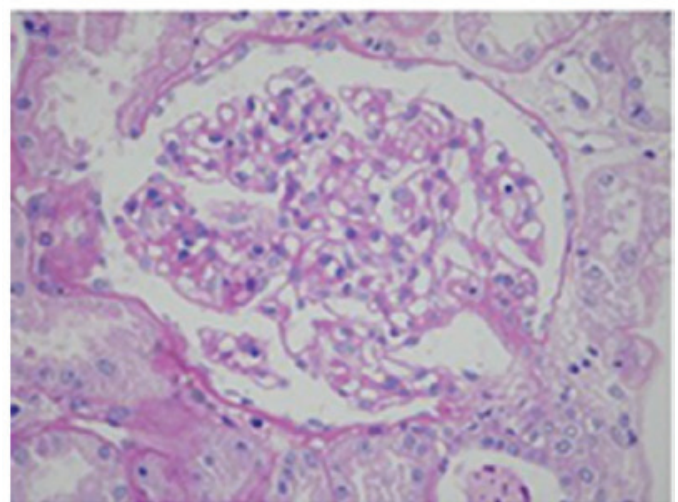

D

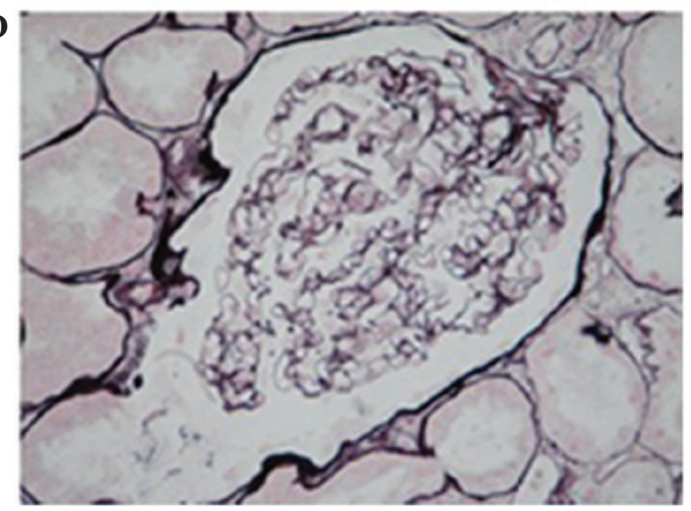

Figure 2. Pathological examination. (A) Hematoxylin and eosin staining revealing partial lumen ectasia and microvilli exfoliation from epithelial cells (magnification, $\mathrm{x} 400$ ). (B and C) PAS revealing slight hyperplasia of glomeruli mesangial cell and matrix (magnification, $\mathrm{x} 400$ ); (D) Periodic Schiff-Methenamine Silver revealing opening of the capillary loop and no apparent basement membrane thicknening (magnification, $\mathrm{x} 400$ ).

serum creatinine levels. However, the patient presented with no clinical manifestations of thrombotic microangiopathy. The patient was pathologically diagnosed with acute renal tubular 
injury, which resulted from the use of acyclovir and renal obstruction.

Clinical manifestations of the patient in the present study suggest that, following the application of antiviral agents, hydration, alkalization of urine, pro-discharge of medication and use of antagonistic agents should be adopted as preventive measures of adverse events following the use of antiviral agents. High-risk populations should be avoided with regards to using such medication. The proportion of drug-induced interstitial nephritis and nephropathy is relatively high in the cases of acute renal failure, which deserves widespread attention and emphasis. The significance of monitoring renal function in hospitalized patients on acyclovir is strongly supported by the present study. In addition, there is strong evidence that acyclovir can cause neurotoxicity in kidney injury, which could further complicate the patient's clinical status (24-26). Early detection, intervention and treatment contribute to the favorable prognosis of acyclovir-induced acute renal injury.

\section{References}

1. Vachvanichsanong P, Patamasucon P, Malagon M and Moore ES: Acute renal failure in a child associated with acyclovir. Pediatr Nephrol 9: 346-347, 1995.

2. Baker DA, Blythe JG and Miller JM: Once-daily valacyclovir hydrochloride for suppression of recurrent genital herpes. Obstet Gynecol 94: 103-106, 1999.

3. Tomori K, Isozumi K, Motohashi S, Komatsumoto S and Fukuuchi Y: A young patient of acute encephalitis complicated with acyclovir encephalopathy without renal dysfunction. Rinsho Shinkeigaku 43: 470-476, 2003 (In Japanese).

4. Fleischer R and Johnson M: Acyclovir nephrotoxicity: A case report highlighting the importance of prevention, detection, and treatment of acyclovir-induced nephropathy. Case Rep Med 2010: 602783, 2010.

5. Delluc A, Mocquard Y, Latour P and Goas JY: Encephalopathy and acute renal failure during acyclovir treatment. Rev Neurol (Paris) 160: 704-706, 2004 (In French).

6. Linssen-Schuurmans CD, van Kan EJ, Feith GW and Uges DR: Neurotoxicity caused by valacyclovir in a patient on hemodialysis. Ther Drug Monit 20: 385-386, 1998.

7. Asahi T, Tsutsui M, Wakasugi M, Tange D, Takahashi C, Tokui K, Okazawa S and Okudera H: Valacyclovir neurotoxicity: clinical experience and review of the literature. Eur J Neurol 16: 457-460, 2009.

8. Adair JC, Gold M and Bond RE. Acyclovir neurotoxicity: Clinical experience and review of the literature. South Med J 87: 1227-1231, 1994.

9. Obada EN, Level G, Mathieu P, Parent X, Gilson B and Bindi P: Acute renal failure following a treatment with acyclovir. Nephrol Ther 6: 125-127, 2010.

10. Rao S, Abzug MJ, Carosone-Link P, Peterson T, Child J, Siparksy G, Soranno D, Cadnapaphornchai MA and Simões EA: Intravenous acyclovir and renal dysfunction in children: A matched case control study. J Pediatr 166: 1462-1468.e1-e4, 2015.
11. Rantanen JM, Markvardsen LH, Jakobsen J and Birn H: Acute kidney injury secondary to the first intravenous administration of acyclovir. Ugeskr Laeger 176: pii, 2014 (In Danish).

12. Sodhi PK and Ratan SK: A case of chronic renal dysfunction following treatment with oral acyclovir. Scand J Infect Dis 35: 770-772, 2003.

13. Yavuz BB, Cankurtaran M, Halil M, Dagli N and Kirkpantur A: Renal dysfunction after oral acyclovir treatment in a geriatric woman: A case report. Scand J Infect Dis 37: 611-613, 2005.

14. Becker BN, Fall P, Hall C, Milam D, Leonard J, Glick A and Schulman G: Rapidly progressive acute renal failure due to acyclovir: Case report and review of the literature. Am J Kidney Dis 22: 611-615, 1993.

15. Sagawa N, Tsurutani Y, Nomura K, Okuyama T, Kondo M, Sata A, Miyao M and Mizuno Y: Acyclovir-induced neurotoxicity and acute kidney injury in an elderly diabetic patient treated with valacyclovir: report of a case. Nihon Ronen Igakkai Zasshi 51: 581-585, 2014 (In Japanese).

16. Lam NN, Weir MA, Yao Z, Blake PG, Beyea MM, Gomes T, Gandhi S, Mamdani M, Wald R, Parikh CR, et al: Risk of acute kidney injury from oral acyclovir: A population-based study. Am J Kidney Dis 61: 723-729, 2013.

17. Seedat A and Winnett G: Acyclovir-induced acute renal failure and the importance of an expanding waist line. BMJ Case Rep 2012: pii, 2012.

18. Obada EN, Level G, Mathieu P, Parent X, Gilson B and Bindi P: Acute renal failure following a treatment with acyclovir. Nephrol Ther 6: 125-127, 2010 (In French).

19. Sawyer MH, Webb DE, Balow JE and Straus SE: Acyclovir-induced renal failure. Clinical course and histology. Am J Med 84: 1067-1071, 1988.

20. Firat H, Brun P, Loirat C and Jacqz-Aigrain E: Kidney failure induced by administration of acyclovir. Apropos of 2 cases. Arch Fr Pediatr 49: 641-643, 1992 (In French).

21. Vashishtha AK and Kuchta RD: Effects of Acyclovir, Foscarnet, and Ribonucleotides on Herpes Simplex Virus-1 DNA Polymerase: Mechanistic Insights and a Novel Mechanism for Preventing Stable Incorporation of Ribonucleotides into DNA. Biochemistry 55: 1168-1177, 2016.

22. Perazella MA: Crystal-induced acute renal failure. Am J Med 106: 459-465, 1999.

23. Mulay SR, Evan A and Anders HJ: Molecular mechanisms of crystal-related kidney inflammation and injury. Implications for cholesterol embolism, crystalline nephropathies and kidney stone disease. Nephrol Dial Transplant 29: 507-514, 2014.

24. De Deyne S, De la Gastine B, Gras G, Dargère S, Verdon R and Coquerel A: Acute renal failure with acyclovir in a 42-year-old patient without previous renal dysfunction. Rev Med Interne 27: 892-894, 2006 (In French).

25. Genc G, Ozkaya O, Acikgöz Y, Yapici O, Bek K, Gülnar Sensoy S and Ozyürek E: Acute renal failure with acyclovir treatment in a child with leukemia. Drug Chem Toxicol 33: 217-219, 2010.

26. Riba Solé M, Farré Riba R, Badell Serra I and Mangues Bafalluy MA: Acyclovir-induced acute renal failure in a paediatric oncology patient. Farm Hosp 35: 281-282, 2011 (In Spanish). 